\title{
Clinical Presentations of Pediatric Horner Syndrome
}

\author{
Frankie K. Smith', Geetanjali Rathore ${ }^{2,3}$, Donny W. Suh ${ }^{2,4}$ \\ ${ }^{1}$ University of Nebraska Medical Center, Omaha, USA \\ ${ }^{2}$ Children's Hospital and Medical Center, Omaha, USA \\ ${ }^{3}$ Department of Pediatrics and Neurology, University of Nebraska Medical Center, Omaha, USA \\ ${ }^{4}$ Department of Ophthalmology and Visual Sciences, University of Nebraska Medical Center, Omaha, USA \\ Email:grathore@unmc.edu
}

How to cite this paper: Smith, F.K., Rathore, G. and Suh, D.W. (2017) Clinical Presentations of Pediatric Horner Syndrome. Open Journal of Ophthalmology, 7, 37-43.

https://doi.org/10.4236/ojoph.2017.71006

Received: January 5, 2017

Accepted: January 31, 2017

Published: February 3, 2017

Copyright (c) 2017 by authors and Scientific Research Publishing Inc. This work is licensed under the Creative Commons Attribution International License (CC BY 4.0).

http://creativecommons.org/licenses/by/4.0/ (c) (†) Open Access

\begin{abstract}
Purpose: To understand the multiple signs of Horner syndrome and to recommend protocols for pediatricians to obtain an accurate diagnosis of Horner syndrome. Methods: The medical records of 17 pediatric patients with Horner syndrome, neonates to eighteen years of age, were collected and analyzed. Data recorded included age, presenting symptoms, other medical history, allergies, medications, pupil size, presence of anhidrosis, and presence of ptosis. From the available pupil sizes, average degree of anisocoria was calculated. Results: All 17 patients had other clinical findings of Horner syndrome in addition to anisocoria. On initial evaluation, $100 \%$ had ptosis and $25 \%$ had anhidrosis. Of the available pupil size data, the average level of anisocoria was $2.06 \mathrm{~mm}$, with a standard deviation of $1.17 \mathrm{~mm}$. Conclusion: Physicians are reminded to measure pupil size to determine the degree of anisocoria when present, as it may help distinguish benign conditions from underlying pathology. Educating pediatricians on measurement of anisocoria and additional signs of Horner syndrome will help with proper referral patterns.
\end{abstract}

\section{Keywords}

Horner Syndrome, Anisocoria, Ptosis, Anhidrosis, Pediatric

\section{Introduction}

Anisocoria in children is often an alarming sign due to the potential life threatening etiologies. Horner syndrome, a neuro-ophthalmologic condition caused by a lesion in the oculo-sympathetic pathway, should be considered in these patients. The sympathetic trunk originates in the hypothalamus and caudal spinal cord and continues along the brachial plexus and over the apex of the lungs to 
synapse in the superior cervical ganglion located at the carotid bifurcation [1]. The post-ganglionic fibers then re-enter the skull, ascending within the adventitia of the internal carotid artery to innervate the dilator pupillae and the Mueller's muscle, causing dilation of the pupil and elevating the eyelid. Therefore, a lesion anywhere in the oculo-sympathetic pathway results in ipsilateral miosis and ptosis. The postganglionic fibers also innervate the facial sweat glands, hence if the injury occurs along the path of the carotid, ipsilateral anhidrosis is also seen [2]. Horner's syndrome can be congenital, acquired from trauma/mass lesion or iatrogenic from surgical manipulation. Testing to localize the site of the lesion is recommended to determine the underlying cause [3]. Most newly-diagnosed cases require neuro-imaging, commonly MRI, unless there is evidence of obvious trauma, or if diagnosis occurs after a surgical procedure.

When a patient presents with Horner syndrome, in addition to the classic triad of eyelid ptosis, anhidrosis, and anisocoria, diagnostic signs can include heterochromia irides, straight hair on the affected side with curly hair on the unaffected side, and reverse ptosis [4]. In pediatric patients with Horner syndrome, impaired facial flushing may be more apparent than anhidrosis [5].

Pediatric ophthalmologists receive multiple referrals annually for Horner syndrome evaluation based on the presence of anisocoria alone. Documentation of presence or absence of other associated symptoms and the degree of anisocoria are often not available. The purpose of this study is two-fold: to understand the multiple signs of Horner syndrome and to recommend protocols for pediatricians to obtain an accurate Horner syndrome diagnosis. A majority of pediatric Horner syndrome patients exhibit physical signs in addition to anisocoria. Understanding these additional signs is imperative to determine a referral to an ophthalmologist. Having a better understanding of all of the signs of Horner syndrome could help decrease the number of false positive diagnoses.

\section{Patients and Methods}

The Institutional Review Board (IRB) of Children's Hospital \& Medical Center and University of Nebraska Medical Center in Omaha, Nebraska, approved this study. Retrospective chart review was performed on patients diagnosed with Horner syndrome in the pediatric neurology clinic. 17 patients $<18$ years old met the inclusion criteria. In the chart review for each patient, data including age, presenting symptoms, other medical history, allergies, medications, pupil size, presence of anhidrosis, and presence of ptosis were analyzed and recorded if relevant. We also looked for documentation of additional findings on the exam on the initial pediatric neurology note.

\section{Results}

All 17 patients had a confirmed diagnosis of Horner syndrome (Figure 1) based on clinical findings, neuroimaging studies, and neurologic evaluation; however, 5 of the 17 patients did not have documented anhidrosis or ptosis on the initial primary care evaluation. This was later confirmed after evaluation by a pediatric 


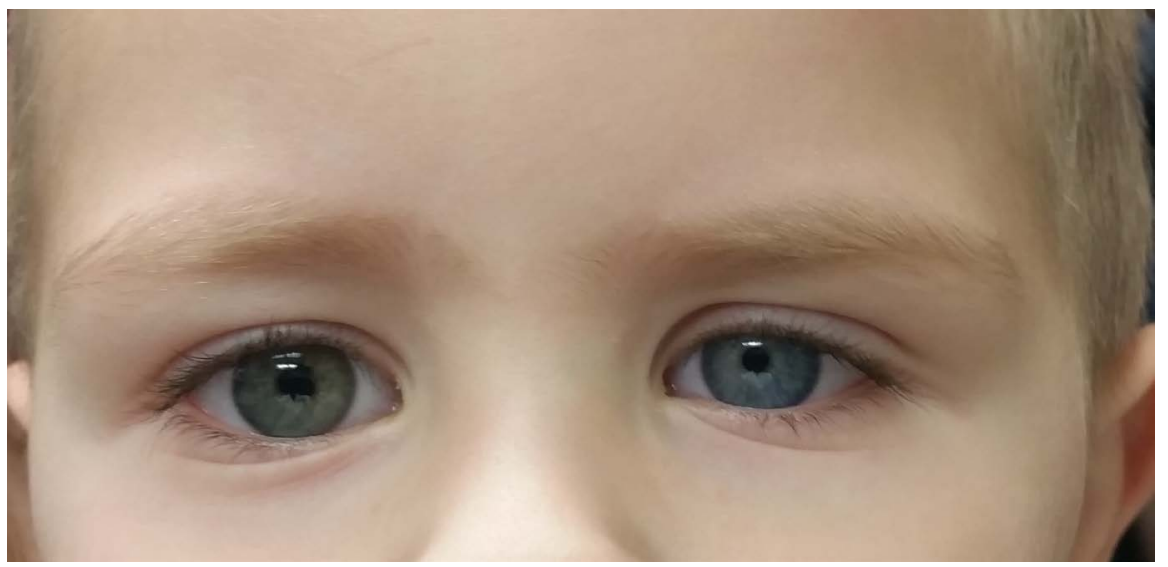

Figure 1. A pediatric patient with Horner syndrome affecting the left eye due to a neuroblastoma. Note the presence of anisocoria with the affected eye being constricted. Patient also has heterochromia and reverse ptosis (elevated lower lid).

neurologist. Of the 12 patients with documentation from the initial primary care evaluation, $100 \%$ had ptosis and $25 \%$ had anhidrosis (Table 1 ).

Of the 17 patients diagnosed with Horner's syndrome, no cause was identified in 9 (53\%) of them (idiopathic). 6 (35\%) patients had an identified mass lesion compressing the parasympathetic pathway, 4 of which had an underlying neuroblastoma and 2 had neurofibromas of the nerve sheet. One patient had surgical trauma to the parasympathetic tract following repair of an aortic arch, and another patient had left carotid artery occlusion following an infection.

Pupil measurements were performed using various Plusoptix photoscreening devices or pupil size charts. In the 5 patients with pupil measurements, the average level of anisocoria was $2.06 \mathrm{~mm}$, with a standard deviation of $1.17 \mathrm{~mm}$.

\section{Discussion}

Knowing the classic Horner syndrome triad of ptosis, miosis, and anhidrosis, we found that in patients presenting with anisocoria, ptosis was present in $100 \%$ of the documented cases, and anhidrosis was present in $25 \%$. This is consistent with the study done by Suh et al., in which $100 \%$ of the 32 diagnosed Horner syndrome patients had associated ptosis [4]. In addition, the study by Suh also found that $9.4 \%$ of the patients had anhidrosis [4]. The consistent presence of other signs in addition to anisocoria, especially ptosis, can be critical for an accurate diagnosis of Horner syndrome.

Part of determining an accurate diagnosis is better documentation of clinical findings. In addition to documenting the actual presence of anisocoria, accurate pupil measurement is imperative. There is currently limited data available to physicians detailing the degree of anisocoria that should warrant further evaluation for Horner syndrome. A study conducted by Suh et al. found that in children with no Horner syndrome, over half of the patients studied had physiologic anisocoria up to $0.5 \mathrm{~mm}$ [4]. In patients with Horner syndrome, the majority had anisocoria greater than $1.3 \mathrm{~mm}$ [4]. This study suggested that anisocoria greater than $1.3 \mathrm{~mm}$ is therefore unlikely to be physiologic. Using the level of 
Table 1. For the 17 patients (A-Q), age, presenting symptoms, other medical history, allergies, medications, pupil size, presence of anhidrosis, and presence of ptosis are presented if available. Note that in the anhidrosis and ptosis columns, the presence of these signs refers to whether they were documented by the initial primary care provider.

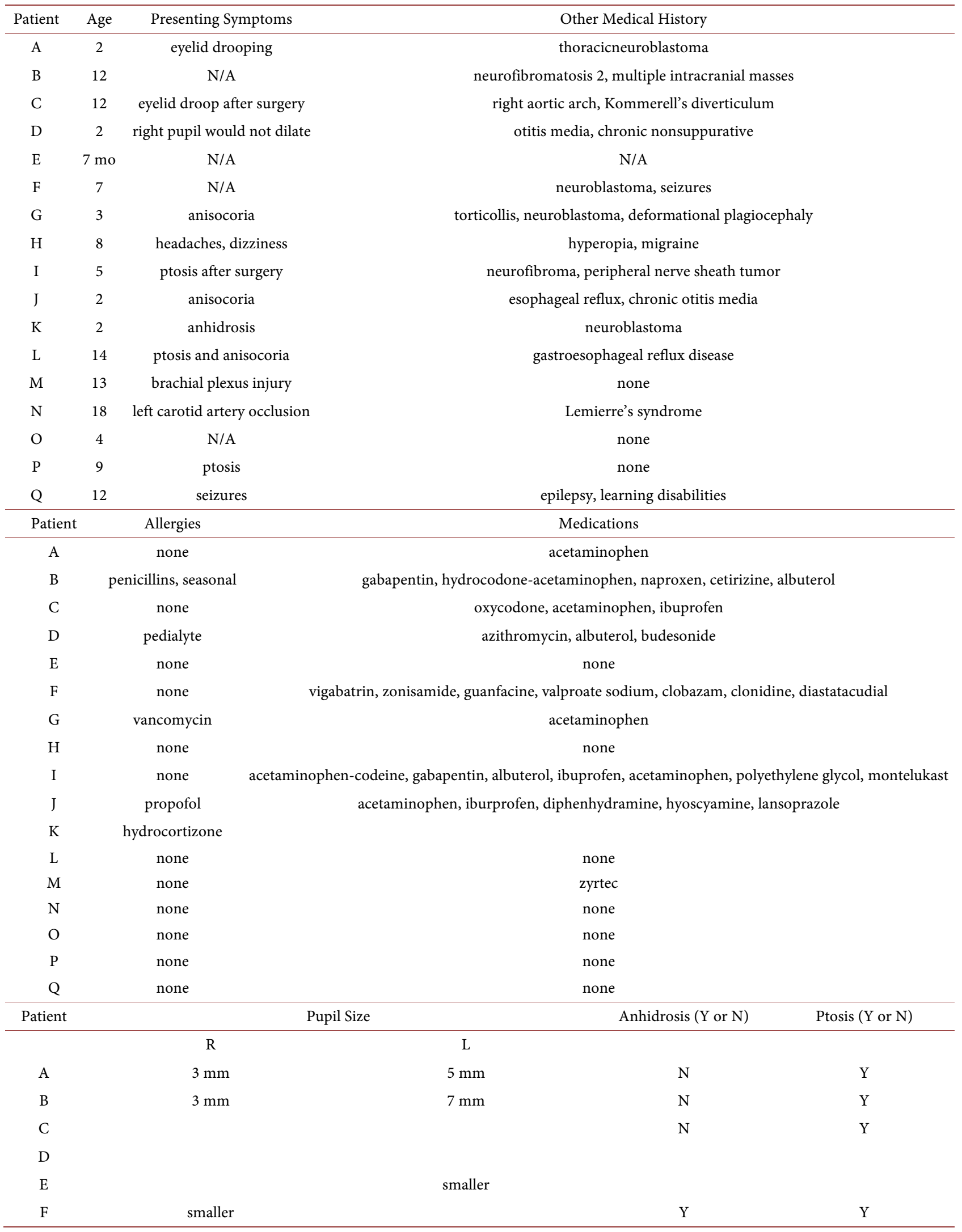




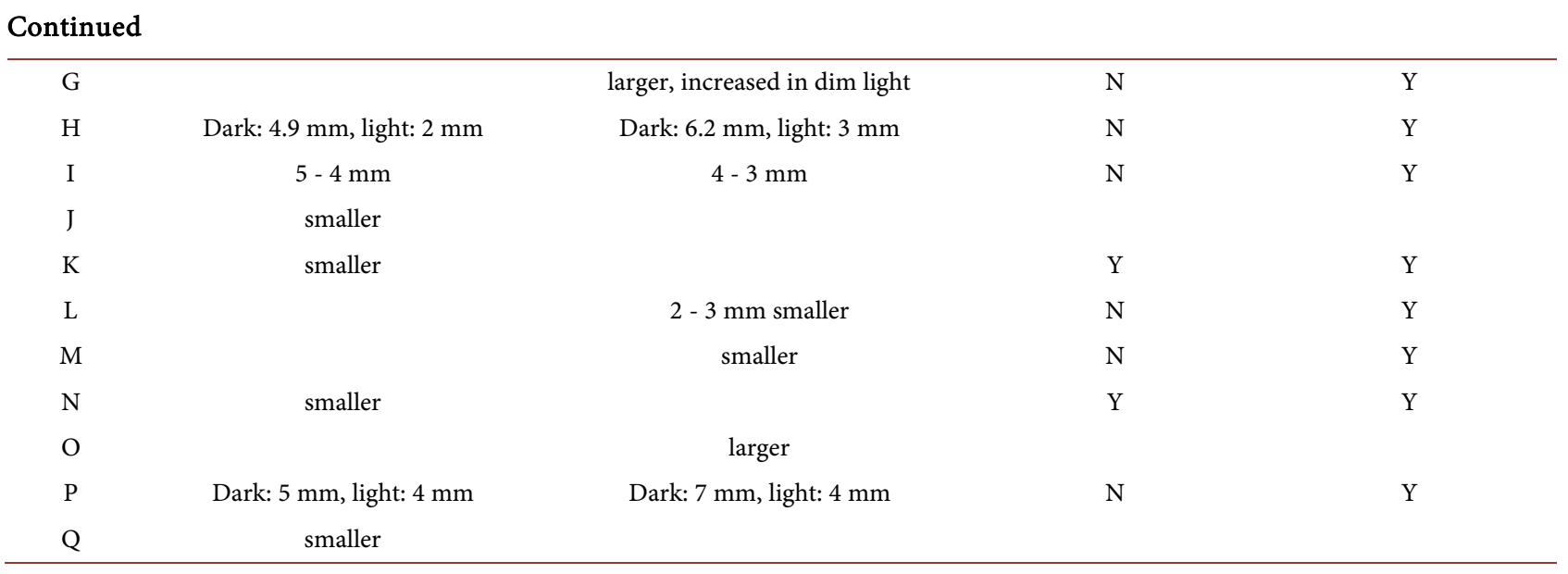

anisocoria can help support a diagnosis of Horner syndrome along with the other classic findings. A non-physiologic level of anisocoria without the other signs of Horner syndrome should be evaluated for other etiologies.

In the chart review for this study, the average level of anisocoria for the 5 Horner syndrome patients with pupil size data available was $2.06 \mathrm{~mm}$. This finding was in accordance with the study by Suh et al. [4]. In addition, given that only five of the patients had pupil size data available, physicians should be reminded to measure pupil size to determine the degree of anisocoria when it is present. While not all physicians have access to the more sophisticated technology used to precisely measure pupil size and anisocoria, pupil sticks, millimeter rulers, and pupil size charts are excellent alternatives. Further research into easily accessible technology that can measure precise pupil size would be beneficial.

In addition to testing pupil size, observations for other signs of Horner syndrome are imperative in diagnosing patients with this condition. Measuring pupil size in bright and dark rooms can help differentiate between Horner syndrome and physiologic anisocoria. Anisocoria associated with Horner syndrome will become more apparent in darkness due to the lack of stimulation to the iris dilator muscle on the affected side. Both pupils should also react normally to light and accommodation given that the iris sphincter muscle is innervated by the parasympathetic pathway. However, affected Horner's pupils will have a dilation lag after room lights are dimmed. Often, the ipsilateral lower lid will be raised, a sign called reverse ptosis.

Horner syndrome can be acquired, congenital, or idiopathic. The most common cause of congenital Horner syndrome is trauma during birth due to injury to the lower brachial plexus [6]. A limitation of our study is that some of the idiopathic cases may be a result of unknown accidental injury to the lower brachial plexus. Acquired Horner syndrome can be caused by the disruption of the sympathetic pathway due to trauma/surgery or by a mass lesion along the pathway. In our study $47 \%$ of patients had acquired Horner's due to an identifiable cause- 6 of whom had a mass lesion and 2 surgical/infectious complications. These findings suggest a significant number of pediatric patients with acquired Horner's have an underlying mass lesion which may need urgent intervention, 
hence the importance of imaging in these patients.

Another differential to consider when anisocoria and ptosis co-exist is third nerve palsy and cavernous sinus syndrome. Documenting a normal oculomotor exam can help rule these out these potential neurological emergencies [1].

\section{Conclusions}

Horner syndrome is a complex condition involving the oculo-sympathetic pathway. All patients in our study diagnosed with Horner syndrome had findings other than anisocoria alone. Knowing that more is involved than a difference in pupil size, a proper diagnosis of Horner syndrome should include evaluation for the presence of anisocoria, the size of pupils both in light and dark illumination, the presence or absence of ptosis on the ipsilateral side and reverse ptosis, and the presence or absence of anhidrosis on the ipsilateral side. Documentation of such positive and negative findings is imperative. Further, educating pediatricians on other signs of Horner syndrome in addition to anisocoria will help with proper referral patterns. As well, a more accurate diagnosis will reduce the number of false positives that result in burdens both for the physicians and the patients.

The study by Suh et al. concluded that anisocoria greater than $1.3 \mathrm{~mm}$ is unlikely to be physiologic [4]. Our study found that the average level of anisocoria for Horner syndrome patients was $2.06 \mathrm{~mm}$, which is in accordance with the Suh study. Our study was limited by the available pupil size data. Future studies that investigate the degree of anisocoria in Horner syndrome patients are warranted to further verify the use of a value to indicate physiologic or pathologic anisocoria so that a diagnosis of Horner syndrome is not missed as this could have devastating consequences.

\section{Acknowledgements}

We would like to thank Adrian Koesters, PhD, Research Editor, UNMC; Linda Morgan, OD, FAAO, Children's Hospital and Medical Center, Omaha, NE; and Sarah Suh, Arizona State University for reviewing the manuscript. We also thank Jamie Lobato for helping with the chart review.

\section{References}

[1] Cahill, J. and Ross, J. (2015) Eye on Children: Acute Work-Up for Pediatric Horners Syndrome. Case Presentation and Review of the Literature. The Journal of Emer gency Medicine, 48, 58-62. https://doi.org/10.1016/j.jemermed.2014.07.041

[2] Kadom, N., Rosman, N., Jubouri, S., Trofimova, A., Egloff, A. and Zein, W. (2015) Neuroimaging Experience in Pediatric Horner Syndrome. Pediatric Radiology, 45, 1535-1543. https://doi.org/10.1007/s00247-015-3341-9

[3] Wilhelm, H. (1998) Neuro-ophthalmology of Pupillary Function: Practical Guidelines. Journal of Neurology, 245, 573-583. https://doi.org/10.1007/s004150050248

[4] Suh, S., Benson, C. and Suh, D. (2016) The Degree of Anisocoria in Pediatric Patients with Horner Syndrome When Compared to Children without Disease. Journal of Pediatric Ophthalmology and Strabismus, 53, 186-189. 
https://doi.org/10.3928/01913913-20160405-07

[5] Morrison, D.A., Bibby, K. and Woodruff, G. (1997) The Harlequin Sign and Congenital Horners Syndrome. Journal of Neurology, Neurosurgery, and Psychiatry, 62, 626-628. https://doi.org/10.1136/jnnp.62.6.626

[6] Jefferey, A., Ellis, F., Repka, M. and Buncic, J. (1998) Pediatric Horner Syndrome. Journal of the American Association for Pediatric Ophthalmology and Strabismus, 2, 159-167. https://doi.org/10.1016/S1091-8531(98)90008-8

Submit or recommend next manuscript to SCIRP and we will provide best service for you:

Accepting pre-submission inquiries through Email, Facebook, LinkedIn, Twitter, etc. A wide selection of journals (inclusive of 9 subjects, more than 200 journals) Providing 24-hour high-quality service User-friendly online submission system Fair and swift peer-review system Efficient typesetting and proofreading procedure Display of the result of downloads and visits, as well as the number of cited articles Maximum dissemination of your research work

Submit your manuscript at: http://papersubmission.scirp.org/ Or contact ojoph@scirp.org 